\title{
WNK3 Interacts with NCC
}

\author{
Dexuan Wang ${ }^{1,2}$, Shufang $\mathrm{Pan}^{2}$, Yixiao $\mathrm{Xu}^{2}$, Xiaohua $\mathrm{Ye}^{2}$, Xiaobi Ren $^{2}$ \& Qiyi Zeng ${ }^{1 *}$ \\ ${ }^{1}$ Pediatric Center of Zhujiang Hospital, Southern Medical University, Guangzhou, 510282, China; \\ ${ }^{2}$ Department of Pediatrics, The Second Affiliated Hospital \& Yuying Children's Hospital of Wenzhou Medical University, \\ Wenzhou 325000, China
}

Received May 11, 2016; accepted June 17, 2016; published online July 1, 2016

Citation: Wang, D., Pan, S., Xu, Y., Ye, X., Ren, X., and Zeng, Q. (2016). WNK3 Interacts with NCC. Sci China Life Sci 59, 1189-1191. doi: 10.1007/s11427-016-5091-9

\section{Dear Editor,}

NCC (Na-Cl cotransporter) is a cotransporter mainly distributed in the distal tubule of the kidney, functioning to reabsorb sodium and chloride ions from the tubular fluid into the cells of the renal distal convoluted tubule. It is a transmembrane protein belonging to the SLC12 cotransporter family of electro-neutral cation-coupled chloride cotransporters, which is closely related to hypertension (Gamba, 2005). A loss of NCC function can cause Gitelman syndrome, a disease characterized by low blood pressure, hypocalciuria, hypokalemic metabolic alkalosis, and hypomagnesemia, etc. WNK3 (With No Lysine K 3) is highly expressed in the whole length of the renal tubules (Glover et al., 2009). It was previously found to enhance the activity of NCC in Xenopus oocyte (Rinehart et al., 2005). Exploring the regulatory effect of WNK3 on NCC may be helpful to understand the occurrence of abnormal renal sodium transporters and provide new targets for the treatment of hypertension. However, whether WNK3 interacts directly with NCC in mammalian cells still remains unclear. In this article, we report the results of our co-immunoprecipitation experiments and confocal observations, confirming that WNK3 does interact with NCC and that the kinase domain of WNK3 plays an important role in the regulation of NCC.

To explore whether there is reaction between WNK3 and NCC protein, Cos-7 cells were transfected by GFP-NCC with or without HA-WNK3 and co-immunoprecipitation

*Corresponding author (email: Qiyizeng1960@hotmail.com) experiments were performed 48 hours after transfection. As shown in Figure 1, anti-HA antibody with the presence of WNK3 immunoprecipitated NCC whereas serum with the absence of WNK3 did not, suggesting that the WNK3 protein interacts with NCC directly.

In order to investigate the effect of WNK3 on NCC, HA-NCC was transfected into Cos-7 cells while increasing doses of HA-WNK3. Western-blot was carried out with anti-HA or anti-GAPDH antibodies $48 \mathrm{~h}$ later. The NCC protein expression level gradually increased with the increase of WNK3 dosage (Figure S1 in Supporting Information). The expression of NCC and WNK3 was checked under a confocal fluorescent microscope (Figure S2 in Supporting Information). The results showed that WNK3 increased NCC expression both on the cell membrane and in the cytoplasm. It has been confirmed that the enzyme region, the amino terminal, and/or the carboxyl terminal are the key elements of kinase activity. However, whether the enzyme region of WNK3 is necessary in regulating NCC is still unclear. We observed the changes of NCC expression after various WNK3 fragments were transferred into the cells. As shown in Figure S3 in Supporting Information, only the WNK3 fragments containing the enzyme region were able to increase the expression of NCC.

WNK kinases belong to a subfamily of serine/threonine kinases. It was reported that the activity of NCC could be down-regulated by WNK4 (Moniz et al., 2010). Our study showed that in the mammalian cells WNK3 was also a chloride sensitive kinase to NCC expression, with its effect being the opposite of WNK4. And the effect of WNK3 


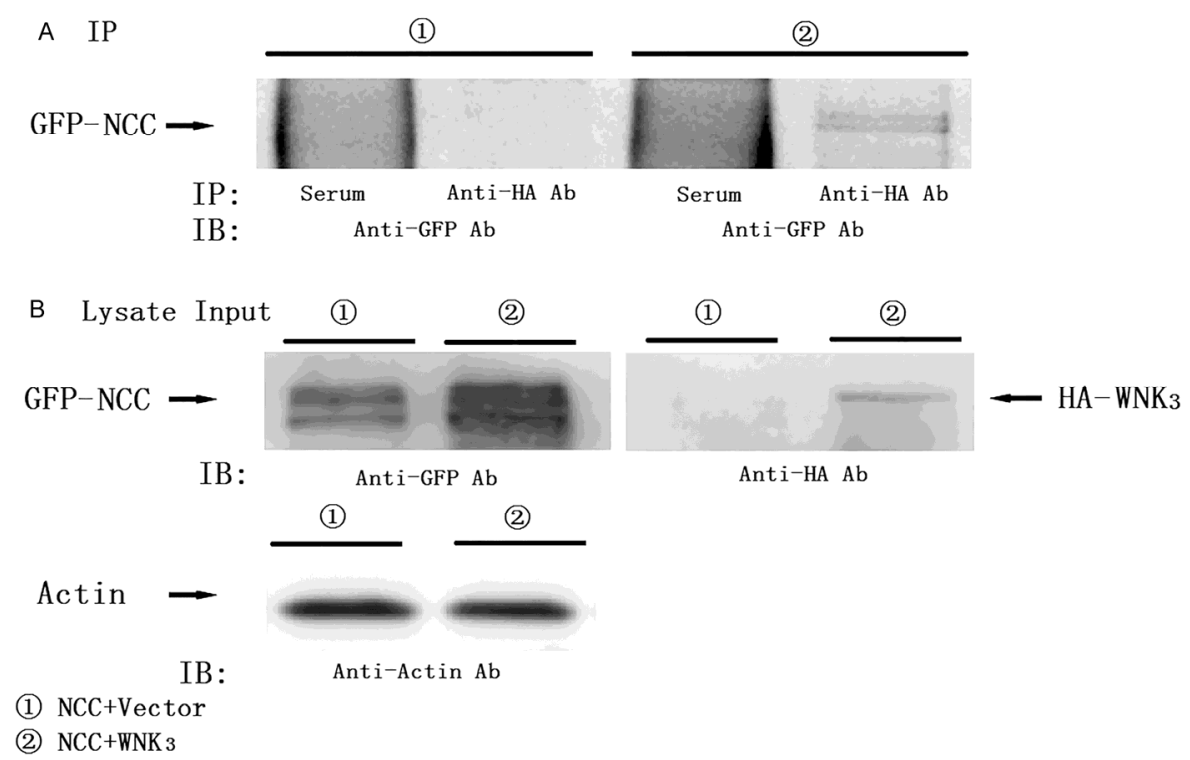

Figure 1 Co-immunoprecipitation between NCC and WNK3. A, Co-IP results. B, WNK3 and NCC protein expression in cell lysates. Cos-7 cells were transfected with GFP-NCC in combination with either HA-Vector or HA-WNK3. $48 \mathrm{~h}$ after transfection, co-immunoprecipitation (Co-IP) experiments followed by western blot analysis were performed. Anti-HA antibody was used to co-immunoprecipitate WNK3. Anti-GFP and anti-HA antibodies were used to probe their respective proteins. Data shown here suggest that WNK3 was able to immunoprecipitate NCC, whereas HA -Vector and serum did not pull down WNK3.

on up-regulating NCC total protein expression could be gradually enhanced with the increase of WNK3 DNA dosage, indicating that this effect is dose dependent. However, the mechanism why WNK3 and WNK4 have opposite effects on NCC remains unclear. Commonly, WNK3 may affect the function of NCC in the following two ways. On one hand, WNK3 can regulate the expression of NCC on the cell surface; and on the other hand it may also influence the function of NCC on the cell membrane by phosphorylation (Ponce-Coria et al., 2008). Our observation after immunofluorescence staining showed that normally NCC was mainly distributed on the Cos-7 cell membrane, with a small amount distributed in the cytoplasm (Figure S2 in Supporting Information). WNK3 could increase the expression of NCC both on the membrane and in the cytoplasm of mammalian cells.

Previous studies have indicated the amino terminal of WNK4 is the critical region in the regulation of NCC (Yang et al., 2005). Our present study found WNK3 (421-1800) fragment without the kinase domain had no effect on NCC at all while wild type WNK3 and WNK3 (1-420) fragment, containing the kinase domain, could increase NCC expression obviously. This sharp contrast between different WNK3 fragments revealed that the stimulation of WNK3 to NCC depended on its kinase activity (Figure S3 in Supporting Information), WNK3 regulates NCC through a kinase dependent mechanism. Research has shown WNK3-D294A can activate KCCs and inhibit NKCCs; and these effects could be prohibited by protein phosphatase inhibitor calyculin A and cyclosporine, implying the existence of some kind of phosphatase in the SPAK-WNK3-NCC compound and the formation of SPAK-WNK3-NCC-phosphatase complex (Lagnaz et al., 2014). Our co-immunoprecipitation results in the present study showed that WNK3 interacted with NCC and the interaction between WNK3 and NCC might up-regulate the expression of NCC by recruiting those partners (i.e., SPAK, WNK3, and NCC) to form the same protein complex. These results indicated that the kinase domain of WNK3 played an important role in the activation of SPAK and the subsequent regulation of NCC.

In summary, our present study demonstrates that WNK3 interacts with NCC and increases the NCC expression on the cell membrane and in cytoplasm together. The kinase domain of WNK3 is necessary for WNK3-mediated NCC regulation.

Compliance and ethics The author(s) declare that they have no conflict of interest.

Acknowledgements This work was supported by the National Natural Science Foundation of China (81200513), Qianjiang Talents Project of Technology Office of Zhejiang Province (2011R10049), and Wenzhou Science \& Technology Bureau (H20110014).

Gamba, G. (2005). Molecular physiology and pathophysiology of electroneutral cation-chloride cotransporters. Physiol Rev 85, 423-493.

Glover, M., Zuber, A.M., and O'Shaughnessy, K.M. (2009). Renal and brain isoforms of WNK3 have opposite effects on NCCT expression. J Am Soc Nephrol 20, 1314-22.

Lagnaz, D., Arroyo, J.P., Chavez-Canales, M., Vazquez, N., Rizzo, F., Spirli, A., Debonneville, A., Staub, O., and Gamba, G. (2014). WNK3 
abrogates the NEDD4-2-mediated inhibition of the renal $\mathrm{Na}^{+}-\mathrm{Cl}^{-}$cotransporter. Am J Physiol Renal Physiol 307, F275-F286.

Moniz, S., and Jordan, P. (2010). Emerging roles for WNK kinases in cancer. Cell Mol Life Sci, 67, 1265-1276.

Ponce-Coria, J., San-Cristobal, P., Kahle, K.T., Vazquez, N., Pacheco-Alvarez, D., de Los, H.P., Juarez, P., Munoz, E., Michel, G., Bobadilla, N.A., Gimenez, I., Lifton, R.P., Hebert, S.C. and Gamba, G. (2008). Regulation of NKCC2 by a chloride-sensing mechanism involving the WNK3 and SPAK kinases. Proc Natl Acad Sci USA 105,
8458-8463.

Rinehart, J., Kahle, K.T., de Los, H.P., Vazquez, N., Meade, P., Wilson, F.H., Hebert, S.C., Gimenez, I., Gamba, G., and Lifton, R.P. (2005). WNK3 kinase is a positive regulator of NKCC2 and NCC, renal cation-Cl- cotransporters required for normal blood pressure homeostasis. Proc Natl Acad Sci USA 10, 16777-16782.

Yang, C.L., Zhu, X., Wang, Z., Subramanya, A.R., and Ellison, D.H. (2005). Mechanisms of WNK1 and WNK4 interaction in the regulation of thiazide-sensitive $\mathrm{NaCl}$ cotransport. J Clin Invest 115, 1379-1387.

Open Access This article is distributed under the terms of the Creative Commons Attribution License which permits any use, distribution, and reproduction in any medium, provided the original author(s) and source are credited.

\section{SUPPORTING INFORMATION}

Figure S1 WNK3 increases NCC protein expression. A, Representative immunoblot for HA-NCC and HA-WNK3; B, bar graphs representing the summarized data of all 3 experiments. The ratio of HA-NCC over GAPDH in groups with WNK3 showed statistical significance, in comparison with that of the control group (i.e., the group without WNK3).

Figure S2 WNK3 increases NCC expression on the cell membrane and in cytoplasm. The WNK3 kinase is mainly distributed in the cytoplasm of Cos-7 cell. It increased NCC expression both on the cell membrane and in the cytoplasm under the confocal fluorescent microscope. A, Cos-7 cells transfected with GFP-NCC and HA-tagged Vector. B, Cos-7 cells simultaneously transfected with HA-WNK 3 and GFP-NCC.

Figure S3 WNK3 with kinase domain increased NCC protein expression. HA-NCC combined with different HA-WNK3 fragments or HA-Vector were transfected into the cells. Wild-type WNK3 and WNK3 (1-420) contain a WNK3 kinase domain, but WNK3 (421-1800) is knocked out of that region. $48 \mathrm{~h}$ after transfection, cell lysates were subjected to SDS-PAGE for western blot analysis. Antibodies for HA (for WNK3 and NCC) and GAPDH were used for western blot analysis. A, Representative immunoblot for WNK3 fragments, NCC and GAPDH; B, Bar graphs representing the summarized data of the NCC ratio from groups with different WNK3 fragments. The data showed that wild-type WNK3 and WNK3(1-420) significantly increased NCC expression $(P<0.01)$, while there was no significant difference between WNK3(421-1800) and the HA-Vector groups.

The supporting information is available online at life.scichina.com and link.springer.com. The supporting materials are published as submitted, without typesetting or editing. The responsibility for scientific accuracy and content remains entirely with the authors. 